\title{
Modificaciones Endocrinas Inducidas por la Actividad Física
}

\author{
Dr. Gabriel Uribe E.*
}

Es un hecho conocido que mujeres que practican ejercicio violento o extenuante presentan frecuentemente irregularidades menstruales. Sin embargo, el reconocimiento de este problema, lleva pocos años.

Fue en la década de los setenta cuando las mujeres comenzaron a practicar el deporte en forma masiva y cuando las competencias hasta ese entonces reservadas para los hombres, se programaron para mujeres. Las competencias atléticas han sido cada vez más largas y exigentes. Ahora no es raro ver competir a mujeres junto a los hombres en pruebas de alta resistencia tal como el Triatlon.

A pesar de los múltiples informes en la literatura sobre el tema, da la impresión de que existe confusión en cuanto a la causa de las irregularidades menstruales y muchas dudas en cuanto a su tratamiento.

Endocrinología del niño y el adolescente. Secreción normal de Gonadotropinas

Para entender las modificaciones hormonales en la mujer que practica el ejercicio, es necesario tener algunas nociones sobre el ciclo menstrual normal en la mujer sedentaria.

En el embarazo existen niveles bajos de FSH y LH y progresivamente altos de gonadotropinas, especialmente la gonadotropina coriónica (HCG), (Fig. No. 1) la cual puede llegar a más de 100.000 unidades. La progesterona aumenta progresivamente hasta el final del embarazo cuando alcanza niveles que oscilan entre 100 y $200 \mathrm{ng} / \mathrm{ml}$, siendo la placenta el órgano de mayor producción; unos 250 mgs. por día. Los niveles de estrona y estradiol están

Profesor Titular, Departamento de Ginecología y Obstetricia Facultad de Medicina. Universidad de Antioquia. aumentados unas 100 veces con respecto a las no gestantes. En el feto la producción de sulfato de dehidroepiandrosterona (DHAS) aumenta progresivamente con el aumento del peso de la glándula suprarrenal fetal. El embarazo se comporta como un estado hiperhormonal con relación a la mayor producción de esteroides.

\section{FIGURA 1}

SECRECION DE GONADOTROPINAS EN LA VIDA FETAL, INFANCIA Y PUBERTAD

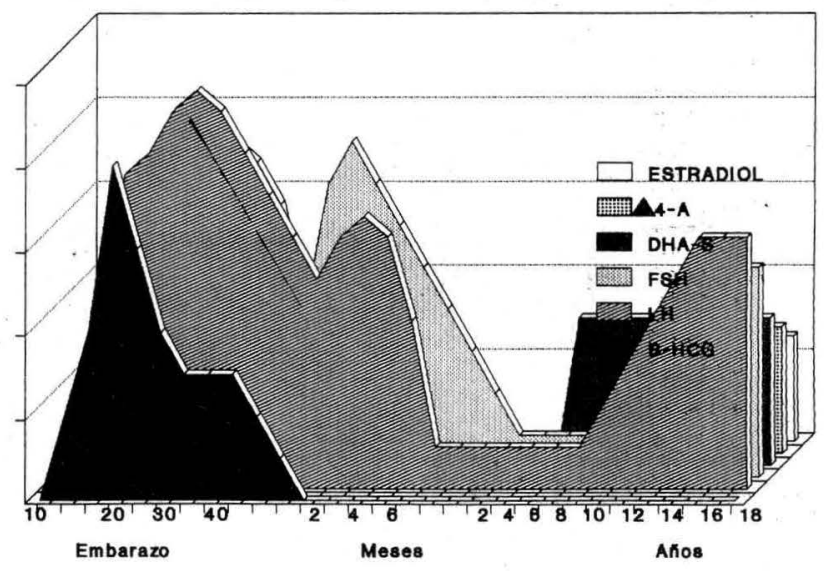

La producción de gonadotropinas durante la vida fetal es bien conocida. Los niveles de hormona folículo estimulante (FSH) y hormona luteinizante (LH) son altos, similares a los existentes en la menopausia. Hay un aumento postnatal de las gonadotropinas, especialmente de FSH hasta los 2 años, para luego descender a su nivel más bajo hasta los 7-8 años cuando se inicia la pubertad.

No se conoce el estímulo preciso que inicia los cambios de la pubertad. La teoría más aceptada es la de que DHAS comienza a aumentar a los 6-8 
años, seguida de la FSH. La pubertad se asocia con el desarrollo de secreción episódica nocturna de LH durante el período del sueño. Luego, por acción sinérgica de FSH y LH se desarrolla el folículo ovárico hasta ocurrir la ovulación y aumentan los esteroides sexuales (estradiol y progesterona).

La menarquia ocurre entonces entre los $11 \mathrm{y}$ 16 años, con un promedio a los 12,8 y es influenciada por factores genéticos, socioeconómicos, geográficos, estado de salud y peso corporal (promedio de 48 Kgms. y un porcentaje de grasa corporal del $17 \%$.)

\section{El ciclo menstrual normal}

Los niveles plasmáticos de estradiol comienzan a aumentar en forma significativa a partir del 7o. día del ciclo, inmediatamente después del proceso de selección del folículo dominante. (Fig. No. 2). Concomitantemente hay una disminución progresiva de los niveles circulantes de FSH. Los niveles de LH estimulan la producción de andrógenos en la teca durante la fase folicular, los cuales se aromatizan a estradiol. Cuando los niveles de estradiol alcanzan 150 a 200 pg. inducen la descarga de FSH y LH en la mitad del ciclo. La LH desencadena la ovulación e inicia la luteinización y la formación del cuerpo lúteo. Estimula la producción de progesterona en las células de la granulosa luteinizadas alcanzando un pico en la mitad de la fase lútea, acompañado unos días después del pico de estradiol. El cuerpo lúteo es una glándula endocrina autolimitada por un proceso luteolítico local, que produce su regresión si no existe embarazo y por lo tanto se produce la menstruación.

\section{FIGURA 2}

\section{CICLO OVARICO NORMAL}

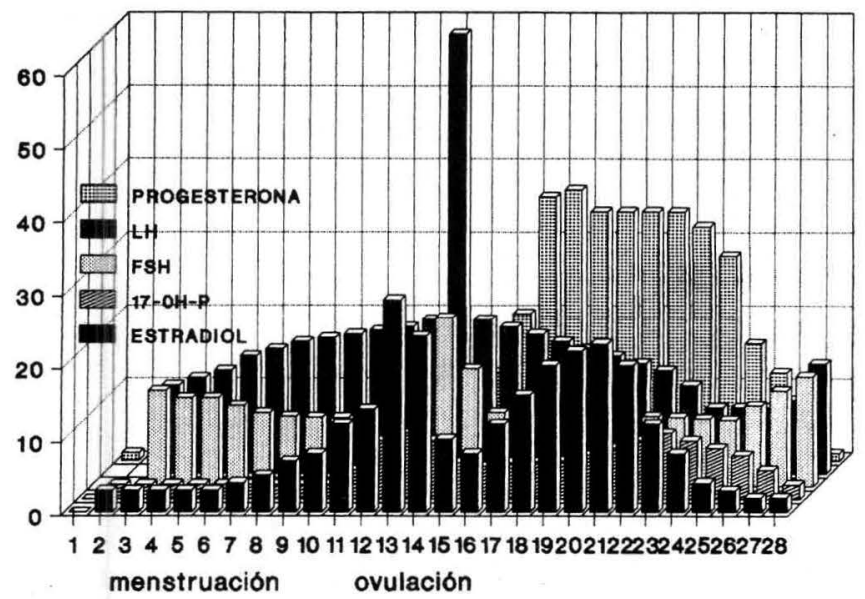

Neuroendocrinología y ciclo menstrual

Repasemos algunos conceptos básicos con respecto a la interacción del cerebro y la ovulación. Se sabe que para que se produzca la ovulación se necesitan los siguientes requisitos:

1. La secreción de GnRH debe ser normal en amplitud y frecuencia (un pulso cada 60 a $90 \mathrm{mi}-$ nutos en la fase folicular).

2. La GnRH actúa sobre la secreción de gonadotropinas a tres niveles:

a. Síntesis y almacenamiento (pool de reserva). b. Activación y movimiento.

c. Liberación inmediata (pool de secreción).

3. Los niveles bajos de estrógenos favorecen la síntesis y almacenamiento de FSH y tienen escaso efecto sobre la secreción de LH.

4. Los niveles elevados de estrógenos desencadenan la descarga de FSH y LH en la mitad del ciclo. Los niveles sostenidos de estrógenos (estro persistente) conducen a una secreción elevada y sostenida de $\mathrm{LH}$, tal como ocurre en el síndrome de ovario poliquístico (SOP).

5. El pico de progesterona preovulatorio (2 a $4 \mathrm{ng} / \mathrm{ml}$ ) potencia el pico estrogénico para la exocitosis masiva de gonadotropinas en la hipófisis (pico de gonadotropinas).

6. Los niveles elevados de progesterona suprimen la secreción hipofisiaria de gonadotropinas al disminuir la pulsatilidad de GnRH en el hipótálamo.

\section{Diversas Teorías}

Se han invocado múltiples teorías para explicar los trastornos menstruales en la mujer sometida al ejercicio. (Fig. 3).

1. Efectos propios del Stress.

2. Porcentajes de grasa corporal.

3. Cambios hormonales a nivel:

a. Hipotalámico - Hipófisis.

b. Suprarrenal.

c. Ovario. 
FIGURA 3

TEORIAS

AMENORREA Y EJERCICIO

\section{1- Efectos propios del Stress}

2- Porcentaje de grasa corporal

3- Cambios a nivel:

a) Hipotálamico-Hipofisiario

b) Suprarrenal

c) Ovarico

\section{Efectos propios del Stress}

Estudios recientes han indicado que los trastornos menstruales en mujeres que hacen ejercicio es el resultado de una disfunción hipotalámica. La arrogancia del atleta, la sensación de euforia y de satisfacción después de un esfuerzo deportivo es consecuencia de la liberación de opiáceos endógenos. Se sabe que el bloqueo de los opiáceos endógenos aumenta la frecuencia y amplitud de la secreción de GnRH. Los opiáceos estimulan la producción de prolactina y de ACTH. Inhiben la producción de FSH, LH y TSH. Ejercen un efecto indirecto sobre la noradrenalina, produciendo su aumento.

Después de un programa de ejercicios intensos aumentan los niveles circulantes de $\beta$-endorfina y $\beta$-lipotropina.

Todos estos cambios hormonales se asemejan a los que se presentan en la anorexia nerviosa. Se encuentran los mismos patrones de dietas estrictas por razones estéticas o por una fijación casi neurótica sobre la alimentación y control de peso. La única diferencia es que las mujeres deportistas son más concientes de su problema y esto las puede ayudar a buscar soluciones.

\section{Porcentaje de grasa corporal}

Las mujeres jóvenes que pesan menos de $48 \mathrm{Kg}$ o pierden más de $4,5 \mathrm{~K}$ durante el ejercicio son las que probablemente desarrollarán más trastornos menstruales. Existe la hipótesis del peso crítico, según la cual la iniciación de la menarquia y la regularidad de la función menstrual requieren del mantenimiento de un peso por encima de un nivel crítico y que se asocie a una cantidad determinada de grasa corporal. A los 16 años de edad, el percentil 10 $\left(\mathrm{P}_{10}\right)$ equivale a $22 \%$ de grasa corporal. El peso mínimo con relación a la estatura necesario para alcanzar la menarquia y llegar al percentil $25\left(\mathrm{P}_{25}\right)$ a la edad de 13 años es de $48 \mathrm{Kg}$ que equivale aproximadamente a un $17 \%$ de la grasa corporal. Una pérdida de peso entre el 10 y el $15 \%$ del peso normal para la estatura, representa una pérdida aproximada de un tercio de la grasa corporal, lo que conduce a un descenso por debajo del límite del $22 \%$ y se puede asociar con amenorrea. La pérdida de la grasa corporal puede llegar a ser tan grande como del $50 \%$ sin que se altere el peso corporal total por remplazo de la grasa en masa muscular magra.

Sin embargo, algunos autores consideran que el porcentaje de grasa corporal no es muy importante. Bergh T. y Lindahl M. en Suecia presentan un estudio en el cual analizan 248 atletas que practican diversos deportes (Fig.No. 4). Estudian el porcentaje de amenorrea que alcanza a un $12 \%$ y de oligomenorrea un $45 \%$. Lo cual es significativamente más alto cuando se compara con un grupo control. Para los autores del estudio los desórdenes menstruales tuvieron mayor relación con la intensidad y duración del ejercicio que con la pérdida de peso o el porcentaje de grasa corporal.

\section{FIGURA 4}

\section{TIPO DE EJERCICIO}

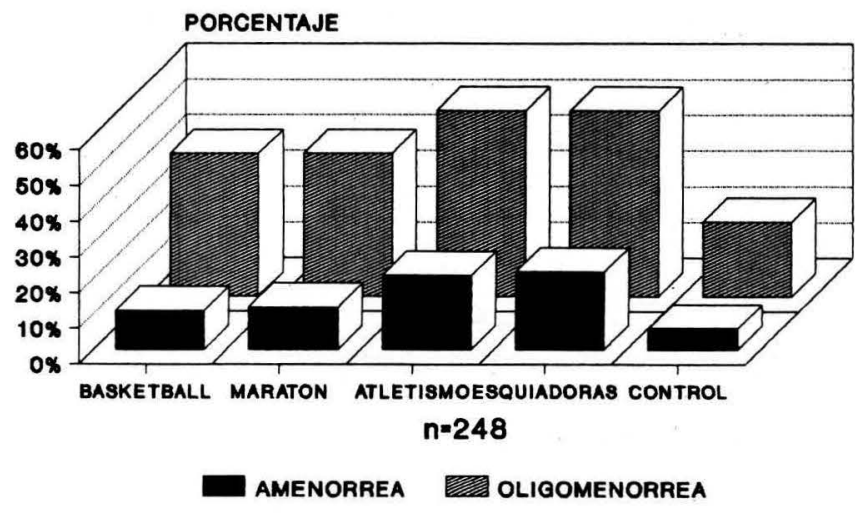

Modificaciones endocrinas a nivel hipotálamo-hipofisis, ovario y suprarrenal

Keizer H. A. y colaboradores, en la Universidad de Limburgo, Holanda, realizaron uno de los trabajos clásicos de determinación de hormonas en mujeres que realizan ejercicio.

En el primer informe estudiaron diez y seis mujeres sedentarias y ocho atletas altamente entre- 
nadas sin problemas ginecológicos previos. Todas se sometieron a una misma actividad física y se les realizó determinaciones hormonales en diferentes períodos de la actividad física.

Los resultados fueron los siguientes:

La concentración de lactato en el plasma aumentó significativamente durante el ejercicio sin que hubiera diferencia estadística entre los grupos (Fig. No. 5).

FIGURA 5

\section{RESPUESTA HORMONAL}

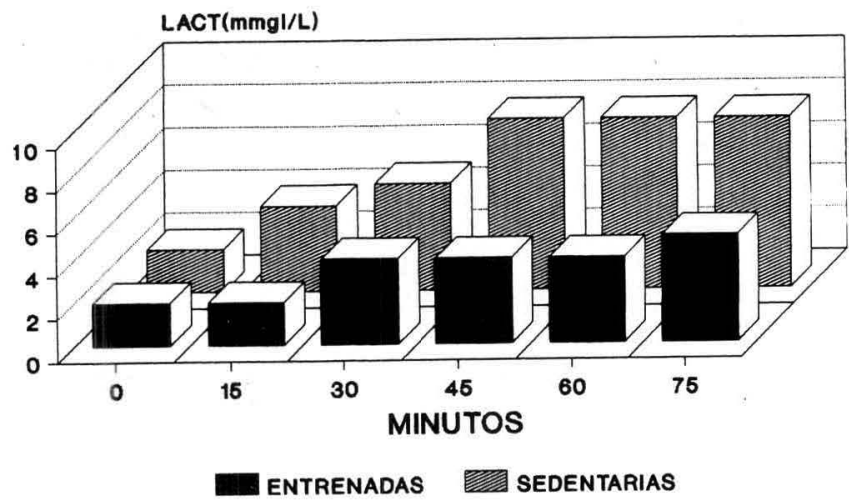

El estradiol y la progesterona aumentaron significativamente en ambos grupos sin existir diferencias entre ambos grupos (Figs. No. 6 y No. 7).

FIGURA 6

\section{RESPUESTA HORMONAL}

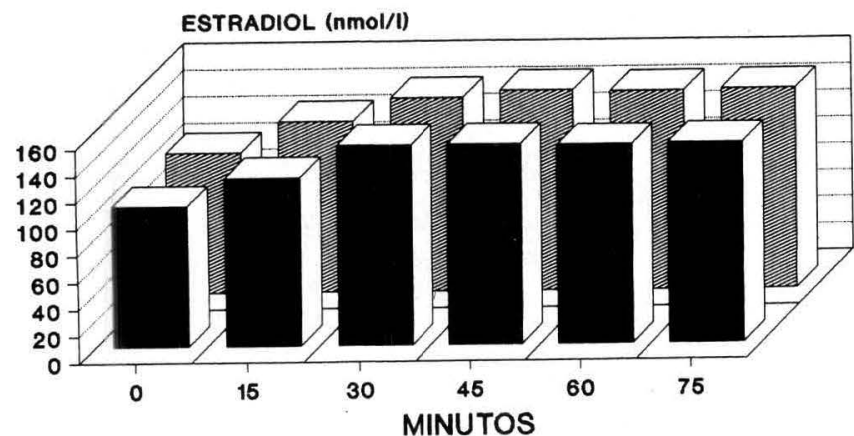

entrenadas pedentarias

La LH y la FSH disminuyeron en ambos grupos, con pequeñas variaciones que no son significativas entre los grupos (Figs. No. 8 y 9). La testosterona aumentó considerablemente en ambos grupos, siendo un poco mayor el aumento en las atletas (Figs. No. 10 y 11 ).
FIGURA 7

\section{RESPUESTA HORMONAL}

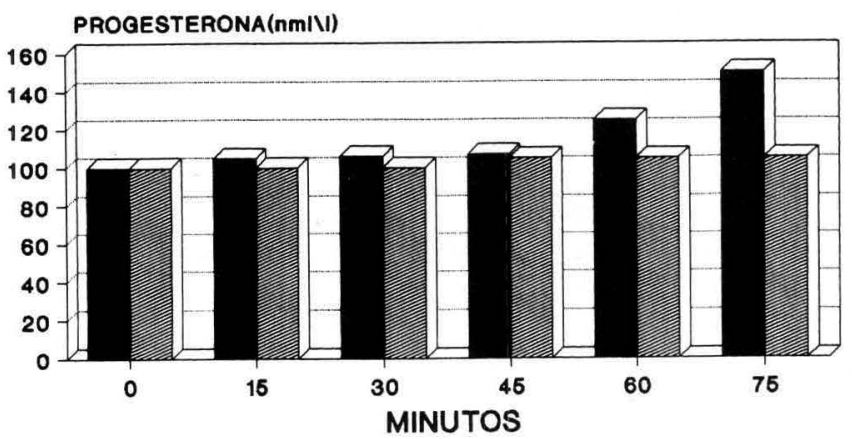

ENTRENADAS YREDTARIAS

FIGURA 8

RESPUESTA HORMONAL

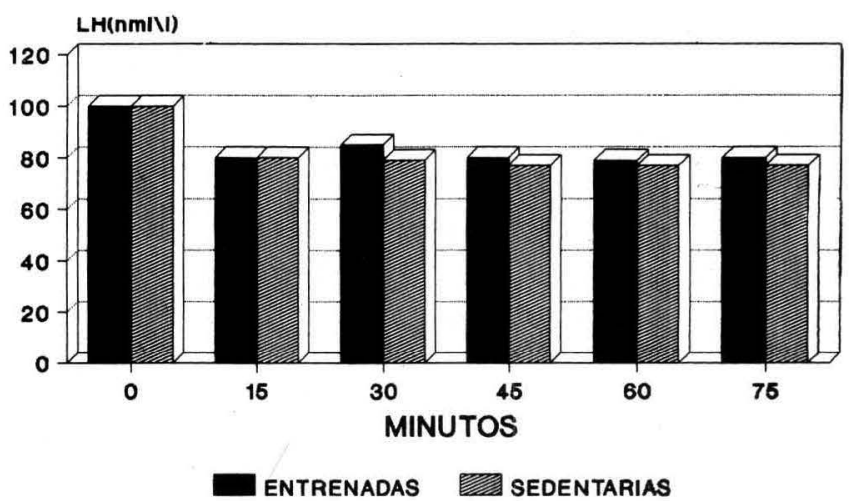

FIGURA 9

RESPUESTA HORMONAL

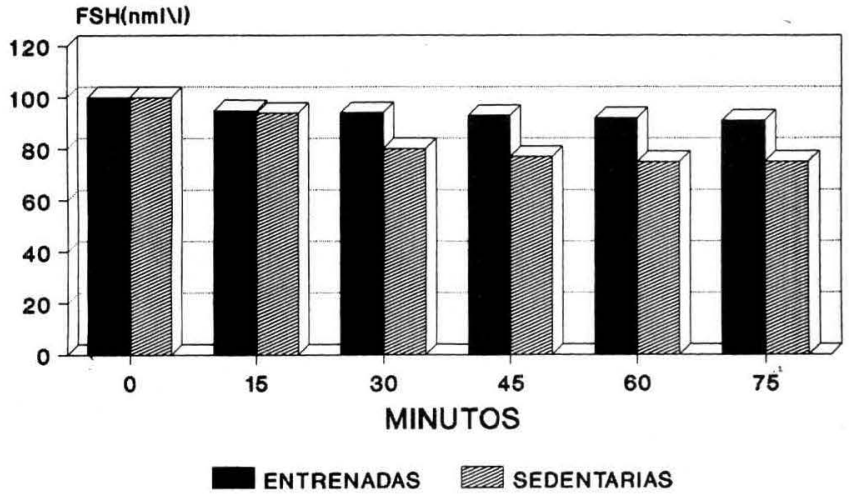

La androstenodiona así como el sulfato de dehidroepiandrosterona (DHEA-S) aumentaron significativamente en ambos grupos, con unos niveles mayores en las sedentarias (Figs. No. 12 y 13 ).

La prolactina aumentó en ambos grupos, sin diferencia significativa entre los grupos (Fig. No.14). 
FIGURA 10

RESPUESTA HORMONAL

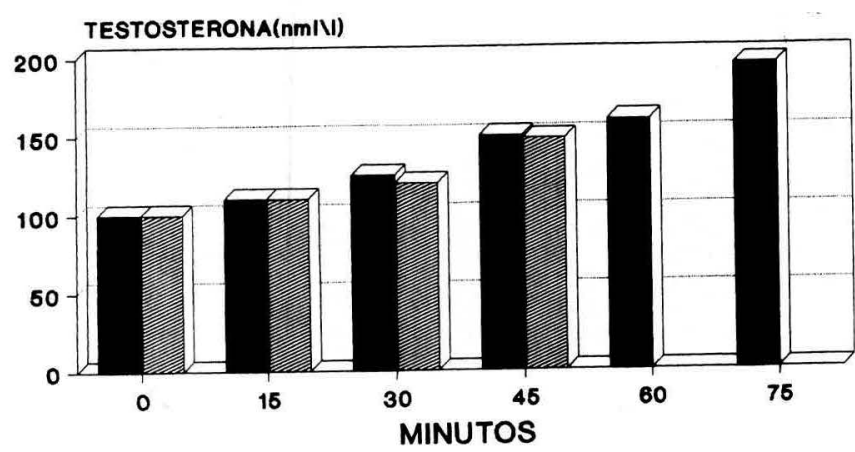

entrenadas sedentarias

FIGURA 11

\section{RESPUESTA HORMONAL}

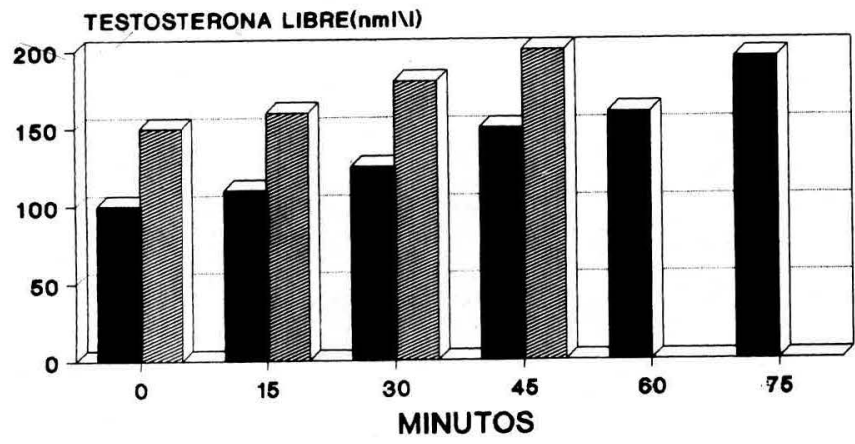

entrenadas sedentarias

FIGURA 12

RESPUESTA HORMONAL

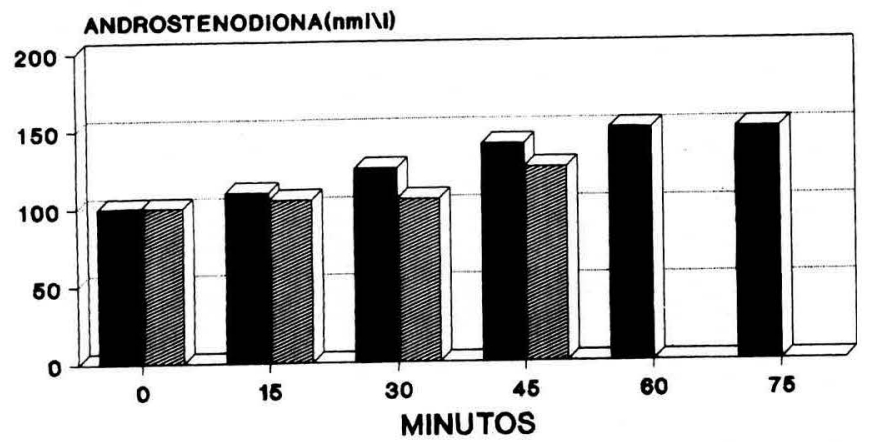

entrenadas Sedentarias

La ACTH aumentó en ambos grupos, pero mucho menos en las no entrenadas (Fig. No. 15).

Como conclusiones de este estudio se tiene:
FIGURA 13

\section{RESPUESTA HORMONAL}

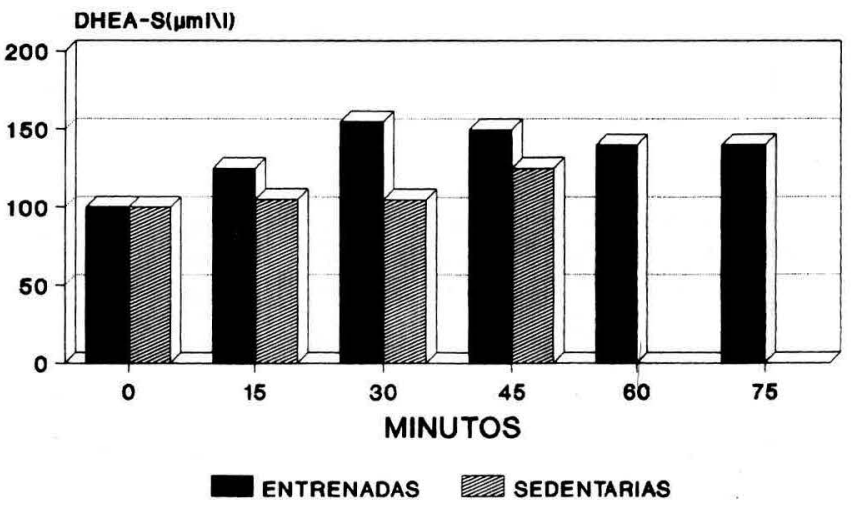

FIGURA 14

RESPUESTA HORMONAL

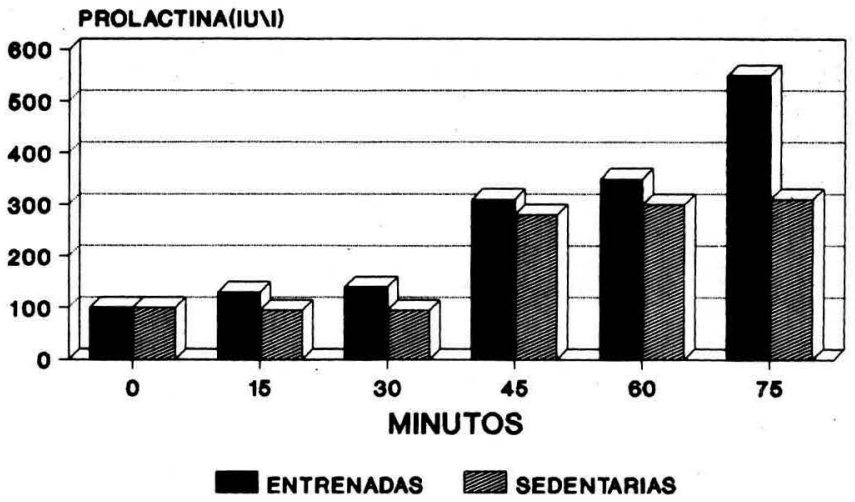

FIGURA 15

RESPUESTA HORMONAL

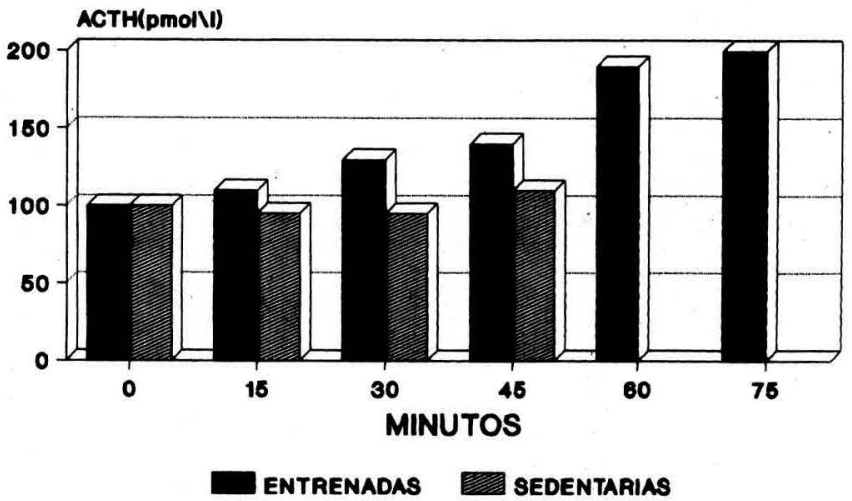

1. Incremento $E_{2}$ y de progesterona asociado a disminución de LH. La explicación de los anteriores fenómenos no es clara. No parece existir un mecanismo específico y parece que esto 
se debe más a una disminución del aclaramiento metabólico.

2. Aumento exagerado de la testosterona debido posiblemente a mecanismos específicos.

3. Aumento de la secreción de DHEA-S debido a una mayor secreción en la suprarrenal.

4. Aumento de ACTH y prolactina como una respuesta al ejercicio y por un posible estímulo hipotalámico.

FIGURA 16

\section{LUEGO DE TRES MESES DE EJERGICIO}

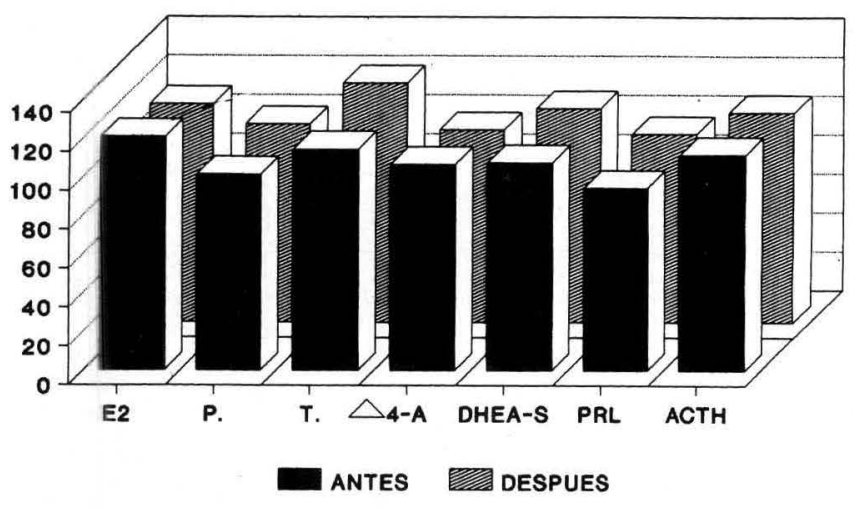

La segunda investigación incluye ocho mujeres entrenadas y controladas antes y después de tres meses de entrenamiento, en donde se midieron las diferentes hormonas y no se apreciaron modificacio. nes que tengan una significancia estadística. Se puede concluir que el entrenamiento intensivo en periodicidad y tiempo sólo produce cambios mínimos en la secreción de esteroides sexuales a nivel ovárico. Los niveles elevados de testosterona y DHEA-S es la respuesta de la glándula suprarrenal a la mayor producción de ACTH secundaria al Stress.

Un tercer informe se basó en el estudio de cinco atletas en fase de entrenamiento a quienes se les realizó diversas pruebas durante la realización de una Maratón.

Los resultados son los siguientes:

1. Los niveles de glicemia se aumentaron en la mayoría de las corredoras, como respuesta a la disminución de la insulina encontrada por otros autores (Fig. No. 17).

2. Aumento del lactato como respuesta a la mayor glicolisis, hallazgo esperado (Fig. No. 18).
FIGURA 17

\section{RESPUESTA HORMONAL DURANTE LA MARATON}

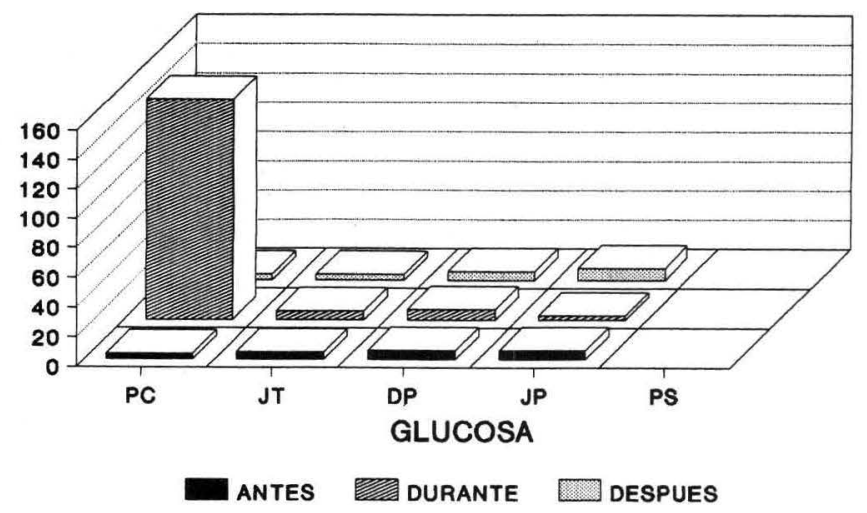

FIGURA 18

\section{RESPUESTA HORMONAL DURANTE LA MARATON}

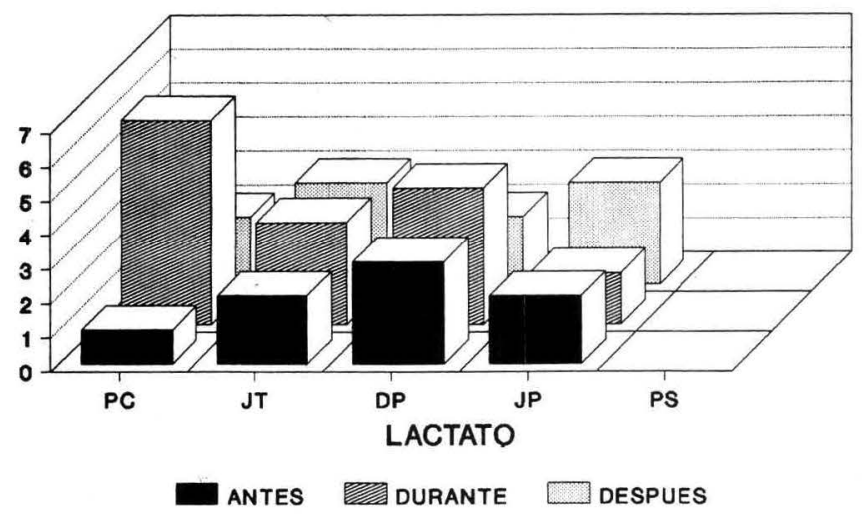

3. Disminución de FSH y $\mathrm{LH}$, en tanto hubo aumento de androstenodiona y DHEA-S (Figs. No.19 y No. 20).

FIGURA 19

\section{RESPUESTA HORMONAL DURANTE LA MARATON}

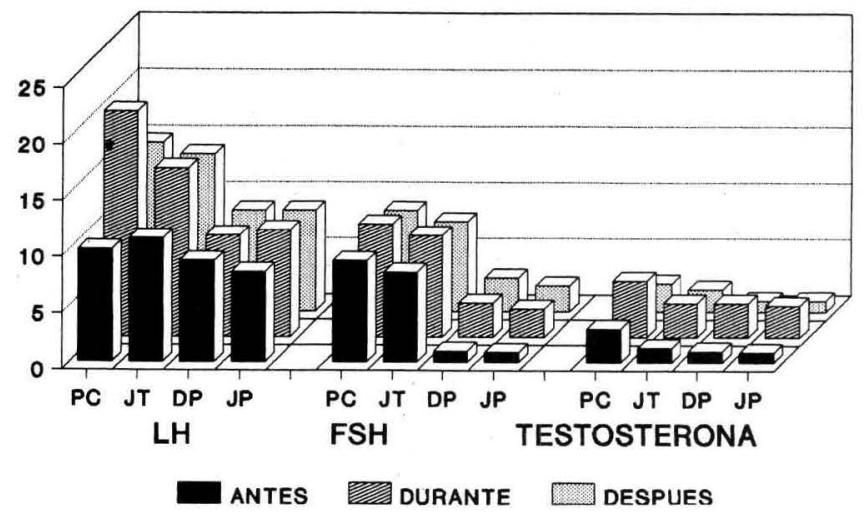


FIGURA 20

\section{RESPUESTA HORMONAL DURANTE LA MARATON}

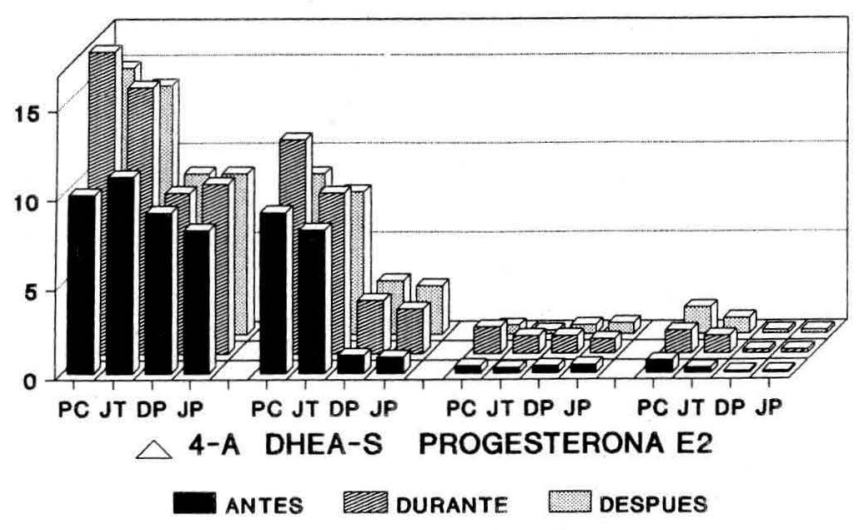

La conclusión más importante de este trabajo, nuevamente, es el aumento significativo de los niveles circulantes de andrógenos y los pocos cambios a nivel del estradiol.

Fisiopatología de la Amenorrea secundaria al Ejercicio

1. Existe aumento en la secreción de los andrógenos suprarrenales (DHEA-S).

2. Aumento de la conversión periférica de prehormonas tal como la transformación por el músculo esquelético de la Androstenodiona en Testosterona. Esta conversión es el resultado del mayor flujo sanguíneo que ocurre a nivel muscular durante el ejercicio.

3. Aumento de los niveles circulantes de prolactina, que podría actuar como estimulador de la secreción de andrógenos suprarrenales.

\section{CONCLUSIONES} finales:

Los autores dan las siguientes conclusiones

1. Los cambios hormonales inducidos por el ejercicio no explican por sí solos la alta incidencia de trastornos menstruales informados en la literatura.

2. Debe ocurrir un sobreentrenamiento para desencadenar modificaciones a nivel del eje hipotálamo-hipófisis.

3. Aumento evidente en la secreción de andrógenos circulantes en las mujeres entrenadas.

\section{Otras causas de Amenorrea}

No todos los casos de amenorrea en atletas se deben explicar por el ejercicio. Es necesario un estudio sistemático de la paciente con el fin de des. cartar las diferentes causas de amenorrea.

1. Fisiológicas: el embarazo y la lactancia.

2. Patológicas:

a. Hipotalámicas: anorexia nerviosa, la seudociesis, ingesta de drogas sicotrópicas, postpíldora.

b. Hipofisiarias: hiperprolactinemia, el síndrome de ovario poliquístico, adenomas, síndrome de Sheehan.

c. Ovárica: menopausia y perimenopausia, menopausia precoz, disgenesia gonadal.

d. Uterina: Síndrome de Asherman.

\section{Diagnóstico}

Para llegar a un correcto diagnóstico se debe realizar una buena historia clínica, haciendo énfasis en el interrogatorio sobre los antecedentes ginecoobstétricos. Se debe practicar un buen examen físico y se pueden realizar algunos procedimientos de consultorio como son la citología funcional y en casos seleccionados, la biopsia de endometrio.

Exámenes de laboratorio

Una vez se ha elaborado una buena historia clínica, el médico está en çapacidad de solicitar los exámenes de laboratorio con el fin de confirmar, el diagnóstico presuntivo. La solicitud indiscriminada de hormonas hacen que el diagnóstico sea muy costoso y poco práctico. Actualmente se pueden solicitar una variedad de hormonas estudiadas por método del radioinmunoanálisis (RIA) tales como:

1. Gonadotropinas

$\mathrm{LH}-\mathrm{FSH}-\mathrm{PRL}-\beta \mathrm{HCG}$.

2. Pruebas tiroideas

TSH, T3, T4.

3. Esteroides gonadales

Estradiol

Testosterona

Androstenodiona 
4. Esteroides adrenales

Cortisol

DHEA-S

17 OH Progesterona

\section{Ejercicio y embarazo}

Por obvias razones, hay pocos estudios en la literatura que nos indiquen los cambios hormonales que suceden durante el ejercicio en la mujer embarazada. Estudios en animales informan como el ejercicio vigoroso aumenta los niveles séricos de catecolaminas dependiendo de la intensidad y duración del mismo. Este aumento desencadena disminución del flujo sanguíneo uterino. En animales inferiores sometidos a ejercicio se ha observado aumento de la mortalidad fetal, desnutrición fetal intrauterina y retraso de la osificación.

Existe unanimidad en recomendar la disminución de la práctica del ejercicio intenso durante el embarazo, especialmente desde la vigésima semana en adelante.

\section{Tratamiento}

Depende del diagnóstico etiológico. Las recomendaciones generales son:

1. Aumento de peso y disminución del ejercicio, conducta que muchas veces no es posible debido a la personalidad e intereses de la atleta.

2. Sustitución hormonal:

Cada vez cobra más importancia la etiología de la osteoporosis en la mujer con amenorrea hipotalámica. Presenta hipoestrogenismo y pérdida de la protección hormonal que favorece la pérdida de la matriz ósea y por lo tanto de calcio. Se recomienda tratar a estas mujeres con estrógenos naturales, 1.25 mgs. del día $1-25$ del ciclo y agregarles 10 mgs de medroxiprogesterona del día 16-25 del ciclo.

3. Si desea un embarazo, debe reducir el ejercicio, aumentar su peso y si hay anovulación, inducir la ovulación con las drogas apropiadas.

4. Si desea planificar usar anticonceptivos orales en dosis bajas.

5. De acuerdo con los cambios hormonales descritos en mujeres que hacen ejercicio, se pudiera sugerir una terapia en base de acetato de ciproterona, un antiandrógeno que tiene acción progestágena, más un estrógeno, procedimiento que bloquearía el aumento de andrógenos y al tiempo se reemplazaría el déficit de estrógenos.

En conclusión, el conocimiento y análisis de los cambios hormonales inducidos por el ejercicio intenso son importantes para la práctica del médico general, el ginecólogo y el especialista en Medicina Deportiva, permitiendo de esta manera hacer un diagnóstico y tratamiento adecuado de estas pacientes.

La niciación de la especialidad en Medicina Deportiva en nuestro país permitirá programar investigaciones en nuestros deportistas con el fin de obtener propias conclusiones.

\section{BIBLIOGRAFIA}

1. SPEROFF L, GLASS RH, KASE NG. Endocrinología ginecológica e infertilidad. 3a. Edición. Ediciones Toray S.A. Barcelona. 1986.

2. LOUCKS AB, HORVATH SM. Athletic amenorrhea: a review, Med. Sci. Sports Exerci. 1: 56-64, 1985.

3. KULPA P. WHITE B. VISSCHER R. Prospective data on moderade exercise during pregnancy - fetal outcome, maternal weight and curse of labor, Med. Sci. Sports Exerci. S46, 1985 .
4. BERGH T., LINDAHL M. Menstrual disturbances in relation to stenuous physical training in adolescent girls, Free communication. XII Congreso Mundial de Ginecología y Obstetricia, Río de Janeiro, 1985.

5. KEIZER HA. Hormonal responses in women as a function of physical exercise and training, Int. J. Sports Med. 1: 137174. 1987. 